\title{
57
}

\section{PEACE: Process Evolution and Adaptation CASE Environment - Its Framework and Requirements}

\author{
Takeshi Yoshida, Atsunori Murata and Motoei Azuma \\ Department of Industrial Engineering and Management, Waseda University, \\ \{yoshi I murata I azuma \}@azuma.mgmt.waseda.ac.jp
}

\begin{abstract}
The PEACE ( Process Evolution and Adaptation CASE Environment ) is a process support environment based on the process management paradigm, the DREAM (Design, Refine, Evolve and $A$ dapt paradigm). The Darwin's theory of evolution and object oriented concept which encapsulates process and product are applied. The environment consists of three sub-systems cooperating to each other to support human agents: the Design and Refinement Sub-system $(D R E S S)$, the Evolution and Adaptation Sub-system (EASS), and the Process Object Management Sub-system (POMSS). The aim of this research is to support designing and managing evolutional and adaptive software life cycle processes, and reduce gigantic human loads caused by diversity of software products and changing requirements. In this paper its concept, framework, and requirements are presented.
\end{abstract}

Keyword Codes: D.2.2; D.2.6: D.2.9

Keywords: Tools and Techniques; Programming Environments; Management

\section{INTRODUCTION}

Various methodologies for software development were proposed. These experiences implies that a good product is produced by a good process. It is widely accepted that the key concept of modern quality assurance is to produce a good product not by testing but by managing the process.

Early studies about software process were based on rigid process model such as [1]. Following the conventional process models, many process models were proposed such as [2]. Recently a focus of software quality management is shifting from products to processes. In spite of great efforts, most of software process stays at premature level, and most software management still depends on human creativity. Process assessment and process improvement require more flexibility because users want to see the interface before designing, and no matter how carefully and deliberately requirements are analyzed, they tend to change. These facts lead to the need of more sophisticated management support environment, which supports manager to improve their processes by evolving and adapting the environment itself to changes in its environment.

\section{DREAM PARADIGM}

The DREAM paradigm (design, refine, $e$ volve, and $a$ dapt paradigm) is a new process paradigm which PEACE environment is based on. Using PEACE a manager can design and manage the evolutional and adaptive software life cycle processes, and reduce gigantic human loads caused by 
diversity of software processes and products. We apply the Darwinian concept and the objectoriented concept to implement three meta-processes.

Design a Process: Because of the fact that a process management still depends on human works, we recognized necessity of designing processes from requirements. We proposed a process design and assessment method [3] in which processes were to be designed based on quality requirement of the software product, and then each process were assessed. We believe that designing a process from requirements can increase quality and productivity.

Refine a Process: While configuration of products are carefully designed and iteratively refined to deal with their diversity, processes are stiffly prepared before process execution. We believe that it makes processes flexible and adaptive to refine the configuration of both processes and products concurrently and iteratively.

Evolve and Adapt a Process: Productivity and quality of products depends on processes. An environment where processes are executed is unstable and changing over time. To obtain better processes it is needed to evolve processes over and over. Through evolving processes the organization can achieve higher productivity and quality of software products and increase and keep competitiveness of the organization.

\section{PEACE ARCHITECTURE}

PEACE environment which are our target and under development consists of three sub-systems (fig.1). Harmony of them and human agents such as manager, developer, and so on enables processes easily designed, enacted, assessed, and improved and human efforts reduced.

DRESS (Design and Refinement Sub-system): DRESS is the Computer Aided Process Design (CAPD) system. Using this human agents can design and refine processes easily. The system contains two facilities; process-object-retrieving facility (PORF) and process-object-browsing facility (POBF). PORF searches the possible process objects which has product attributes similar to those that human agents input from all PO (process object) stored and managed by POMSS. It provides a list of the POs searched for human agents. Human agents can browse POs by POBF. Because POMSS manages POs like class library human agents can track the objects lined to their ancestors and descendent and reach at the objects about which they want to know the information.

EASS (Evolution and $A$ daptation Sub-system): EASS is the Computer Aided Process Improvement (CAPI) system. Using the system human agents can evolve and adapt processes easily. While process improvement now depends on talented persons, this system introduces computational assists to process evolution so it can reduce management loads that manager responsible for process improvement must face. The system includes two facilities; the troublesearching facility (TSF) and the success-searching facility (SSF). TSF searches the POs that was unsuccessfully completed. In contrast the SSF searches the POs finished successfully. Successful process is one that met the process specification and produced the products that met the products requirements. These facilities provides a list of the POs searched.

POMSS (Process Object Management Sub-system): POMSS stores POs designed and refined. During and after enactment experiences that human agents wrote and measured values of process attributes and products attributes are collected and stored by this system. And this provides them to DRESS and EASS when two sub-systems require. In this sub-system POs are handled in class-library manner.

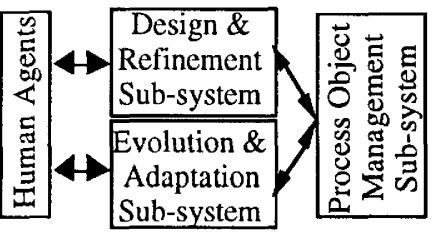

fig.1 PEACE Architecture 


\section{PROCESS IMPLEMENTATION BY PEACE}

In this chapter we explain the method to design, refine, and evolve process by using sub-systems. The procedures for design and refinement of processes is shown in fig.2. The procedures for evolving processes is

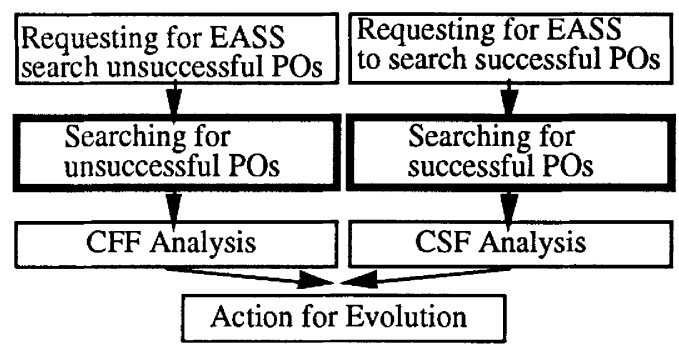

fig.3 Evolving and Adapting a Process

illustrated in fig.3. Bold rectangle in fig. 2 means activity assisted by DRESS. Bold rectangle in fig.3 means activity assisted by EASS. Normal rectangle means human agents' task in both figures.

Designing a Process by DRESS: Human agents such as managers specify product attributes from requirements received. Following it they can request for DRESS to search standard process for the product with specified attributes. If it is not existing one more search is performed by making the abstraction level of object higher. If existing they can use it and tailor it to the products. Following them human agents conforming the searched process to the situation they face.

Refining a Process by DRESS: After searching for standard one human agents refine the process and make process object sub-class(es). This is performed in the case such as menu display design process where similar displays are required but they are slightly different from each other. This situation needs one super-process-object-class and some sub-process-object-class. The refinement of processes does not have to be performed before enactment of the process. It is possibly performed iteratively with product-refinement or enactment of the portion of the process.

Evolving and Adapting a Process by EASS: Human agents can request for EASS to search both successful and unsuccessful objects from all objects stored by POMSS in anytime. Following them they perform critical success factor (CSF) analysis and critical failing factor (CFF) analysis by carefully considering lists of both kinds of objects. Relying the results of analysis they can plan the effective action strategy and can design new standard processes.

\section{CONCLUSION}

Gigantic human loads can be reduced by using PEACE environment. While tasks in process management were depend on human creativity and special talents, some portion of works human agents must face can be assisted by our environment. Especially process improvement activity can be made easier although it has been supported by little tools.

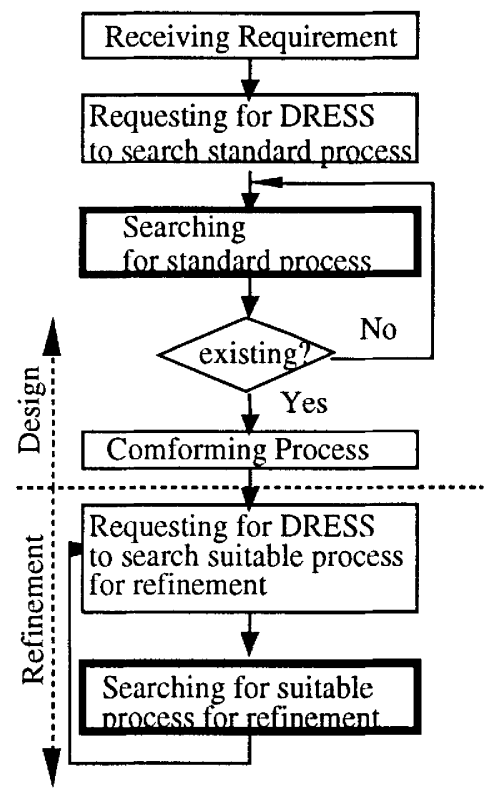

fig.2 Process Design and Refinement 
Software processes can become very flexible and adaptive while they were very stiffly prepared and most process paradigms forced processes to be designed totally before their enactment. This benefit can be produced by encapsulation of process and product.

We mentioned the effects of PEACE but some details should be clarified. They will be presented in further papers.

\section{References}

1. Royce, W.W.: 'Managing the Development of Large Software Systems', Proc. WESCOM, 1970, pp.1-9

2. Boehm, B.W.: 'A Spiral Model of Software Development and Enhancement', Computer, Vol.21, (5), May 1988, pp.61-72

3. Yamaguti, T. and Azuma, M.: 'Quality Management of Software Processes', Proc. 45th Nat'l Conf. of IPSJ, 1992, pp.141-142 (written in Japanese) 